\title{
Compressive Sensing with Optimal Sparsifying Basis and Applications in Spectrum Sensing
}

\section{Citation}

Gwon, Youngjune, H.T. Kung, and Dario Vlah. 2012. Compressive sensing with optimal sparsifying basis and applications in spectrum sensing. Paper presented at IEEE $2012 \mathrm{Global}$ Telecommunications Conference (GLOBECOM 2012), Annaheim, CA, December 3-7, 2012.

\section{Permanent link}

http://nrs.harvard.edu/urn-3:HUL.InstRepos:10000991

\section{Terms of Use}

This article was downloaded from Harvard University's DASH repository, and is made available under the terms and conditions applicable to Other Posted Material, as set forth at http:// nrs.harvard.edu/urn-3:HUL.InstRepos:dash.current.terms-of-use\#LAA

\section{Share Your Story}

The Harvard community has made this article openly available.

Please share how this access benefits you. Submit a story.

Accessibility 


\title{
Compressive Sensing with Optimal Sparsifying Basis and Applications in Spectrum Sensing
}

\author{
Youngjune Gwon, H. T. Kung, and Dario Vlah \\ Harvard University
}

\begin{abstract}
We describe a method of integrating KarhunenLoève Transform (KLT) into compressive sensing, which can as a result improve the compression ratio without affecting the accuracy of decoding. We present two complementary results: 1) by using KLT to find an optimal basis for decoding we can drastically reduce the number of measurements for compressive sensing used in applications such as radio spectrum analysis; 2) by using compressive sensing we can estimate and recover the KLT basis from compressive measurements of an input signal. In particular, we propose CS-KLT, an online estimation algorithm to cope with nonstationarity of wireless channels in reality. We validate our results with empirical data collected from a wideband UHF spectrum and field experiments to detect multiple radio transmitters, using software-defined radios.
\end{abstract}

\section{INTRODUCTION}

Sparse recovery techniques provide a new opportunity to build communication and data-driven systems. We consider compressive sensing (see, e.g., Candès and Tao [1]), a recent theory to extract critical information of data and represent it with substantially reduced measurements of the original data. The simple but effective framework of compressive sensing has enabled a broad range of applications in imaging, data processing in wireless sensor networks [2], cloud computing [3], cognitive radios [4], spectrum sensing [5], and IC Trojan detection [6].

Compressive sensing has three important properties. First, the encoding is blind to the content of a signal (or data) and has low computational complexity suitable for fast, real-time usage. Secondly, the number of measurements required for exact recovery is approximately proportional to sparsity of the signal, not its size. Lastly, the decoding is adaptive in the sense that the quality of recovered data can improve under a fixed number measurements - or equivalently, the required number of measurements that achieves the same quality can decreasewhen a more effective sparsifying basis becomes available. Therefore, an algorithm to find an optimal basis would be critical for performance improvement.

In this paper we propose a method to integrate the finding and the use of optimal sparsifying bases into compressive sensing to lower the number of measurements without affecting the accuracy of decoding. We demonstrate the benefit of our approach with an application system for spectrum sensing. The principle behind our method is Karhunen-Loève Transform (KLT) [7], a classical procedure that reveals the correlation structure of a signal. KLT is optimal such that it decorrelates the signal into a representation comprising only statistically non-redundant coefficients. Thus we can use KLT to derive sparsifying basis that can improve the sparse recovery process of compressive sensing.

Despite the optimal sparsification, KLT has a well-known drawback for being data-dependent. That is, when the correlation structure of a signal changes, the KLT basis also has to be updated. This has been a prohibitive limitation for KLT in realistic settings, where the KLT basis is computed numerically from a sample covariance matrix of training data sets. It is contrasted to an approach based on a fixed support set such as Discrete Fourier Transform (DFT). The recovery with DFT as the sparsifying basis is suboptimal for a given signal, but it is popularly used in compressive sensing because it does not require any data-dependent adaptation.

A novelty in our approach is to recover KLT bases from random projections of a signal, as we normally do to recover the signal with compressive sensing. This allows us to update the KLT basis incrementally from the measurements on the original signal with relatively few measurements.

We summarize the main contributions of this paper concerning the use of optimal bases in compressive sensing: 1) we show a drastic reduction in required measurements when an optimal KLT basis, as opposed to DFT or DCT, is used for decoding; 2) we formulate an online algorithm to update the KLT basis directly from the measured input signal via compressive sensing, which only requires a nonadaptive, fixed support set for recovery (e.g., DFT basis); 3) we show how the previous two work together, using the KLT basis to recover the signal and the DFT to update the KLT basis. Although the latter requires more measurements, it needs not take place frequently; and 4) we demonstrate our approach lead to an overall gain in performance of spectrum analysis using multiple software-defined radios (USRPs) [8] and empirical data measured in the field.

The rest of this paper is organized as follows. In Section II, we review compressive sensing and KLT. Section III introduces a compressive spectrum analysis system. In Section IV, we explain how to compute KLT basis with compressive sensing. Section V presents the CS-KLT online algorithm and discusses our implementation with USRPs. We evaluate the performance of our approach in Section VI and discuss related work in Section VII. Section VIII concludes the paper.

\section{BACKGROUND}

\section{A. Compressive Sensing}

Compressive sensing exploits a sparse structure (either exposed or hidden) in data. Some data naturally exist as a 
sparse form, and most of the others can be made sparse so that only several largest coefficients in some basis-which we call sparsifying basis-suffice to represent the data (e.g., Fourier transform of sinusoids). The fundamental premise of compressive sensing is that we can directly encode all significant coefficients without completely analyzing the data. More importantly, the encoding does not require any prior knowledge on sparsification of the data. Traditional compression schemes such as entropy coding, however, require statistical analysis of the data. Compressive sensing combines the measurement and compression of the data into one-non-analytical, lowcomplexity encoding based on matrix-vector multiplications using a randomly generated measurement matrix.

Consider a vector $\mathrm{x} \in \mathbb{C}^{N}$ and an $M \times N$ measurement matrix $\boldsymbol{\Phi}$ for $M \ll N$. Compressive sensing encodes $\mathbf{x}$ into compressive measurements $\mathbf{y}=\boldsymbol{\Phi} \mathbf{x}$, an $N$ to $M$ reduction in size. The encoding takes place without the knowledge of a transform $\Psi$ (an $N \times N$ matrix), which sparsifies $\mathrm{x}$ such that in $\mathbf{s}=\mathbf{\Psi} \mathbf{x}$ there are only $K \ll N$ nonzero elements (i.e., $\mathbf{x}$ is $K$-sparse). Compressive sensing forms an underdetermined system of equations susceptible to many solutions for $\mathrm{x}$ :

$$
\min _{\mathbf{s} \in \mathbb{R}^{N}}\|\mathbf{s}\|_{\ell_{1}} \quad \text { subject to } \quad \mathbf{y}=\mathbf{\Phi} \mathbf{x}=\left(\mathbf{\Phi} \Psi^{-\mathbf{1}}\right) \mathbf{s}
$$

The Robust Uncertainty Principle [9] states that $M$ needs to be at least $c K \log \frac{N}{K}$ for some small constant $c>0$ for exact recovery. Also, the Restricted Isometry Property (RIP) [10] of $\Phi$ can guarantee a unique solution with high probability by the $\ell_{1}$-norm minimization decoding of $\mathbf{s}$. Linear programming can be used to solve Eq. (1). Once $\mathbf{s}$ is recovered, $\mathbf{x}$ can be determined from $\mathbf{x}=\boldsymbol{\Psi}^{-\mathbf{1}} \mathbf{s}$.

The quality of decoding depends on $M$. More interestingly, the data recovery is incremental-using a smaller $M$ (than required for exact solution) does not disqualify the decoded result entirely. The corresponding largest components recovered (specific to $M$ ) would still be approximately accurate. In other words, the majority of decoding error is contributed by the unrecovered components, and the sum of their magnitudes quantifies the approximate error. If desired accuracy is not met, one can increase $M$ accordingly. Fixing $M$, however, by no means fixes the decoding accuracy because the use of a better sparsifying basis for the data can still improve the performance. Estimating an optimal basis and using it for decoding is the approach we take in this paper.

\section{B. Karhunen-Loève Transform (KLT)}

But how can we systematically find an optimal basis? Consider $\mathbf{x} \in \mathbb{C}^{N}$, a complex-valued, wide-sense stationary signal with mean zero (for simplicity). The covariance matrix of $\mathrm{x}$ can be computed numerically: $\mathbf{R}_{\mathbf{x}}=\mathbb{E}\left[\mathbf{x x}^{\mathbf{H}}\right]$, where the superscripted $\mathbf{H}$ denotes Hermitian transpose (i.e., $\mathbf{x}^{\mathbf{H}}=$ $\left.\mathbf{x}^{* \mathbf{T}}\right) . \mathbf{R}_{\mathbf{x}}$ is real and symmetric, and the eigen-decomposition, $\mathbf{R}_{\mathbf{x}}=\mathbf{Q} \mathbf{\Lambda} \mathbf{Q}^{\mathbf{H}}$, gives columns of $\mathbf{Q}$ the eigenvectors of $\mathbf{R}_{\mathbf{x}}$ and $\boldsymbol{\Lambda}$ a diagonal matrix of the eigenvalues. $\mathbf{Q}$ is an orthogonal matrix, thus $\mathbf{Q}^{-1}=\mathbf{Q}^{\mathbf{H}}$.

The representation $\mathbf{s}=\mathbf{Q}^{\mathbf{H}} \mathbf{x}$ is known as KarhunenLoève Transform (KLT) [7] of $\mathrm{x}$, and we call $\mathrm{Q}$ the KLT

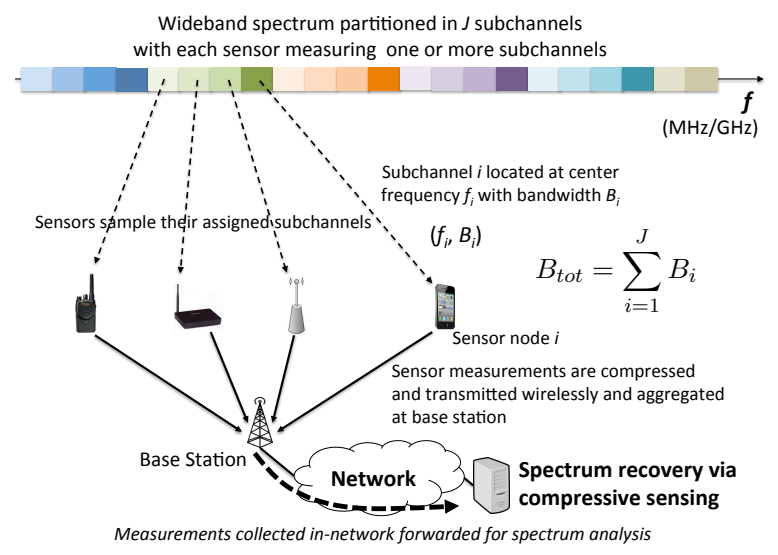

Fig. 1. In this system model, multiple distributed sensor nodes sample their assigned portion of the target spectrum and transmit compressive measurements in-network.

basis or matrix. The covariance matrix of $\mathbf{s}$ is diagonal: $\mathbf{R}_{\mathbf{s}}=\mathbb{E}\left[\mathbf{s ~ s}^{\mathbf{H}}\right]=\mathbb{E}\left[\mathbf{Q}^{\mathbf{H}} \mathbf{x x}^{\mathbf{H}} \mathbf{Q}\right]=\mathbf{Q}^{\mathbf{H}} \mathbf{R}_{\mathbf{x}} \mathbf{Q}=\boldsymbol{\Lambda}$. Thus KLT of $\mathbf{x}$ results in an uncorrelated representation $\mathbf{s}$, whose covariance matrix has zero cross-correlation terms. In other words, $\mathbf{s}$ fully describes $\mathbf{x}$ without any statistical redundancy.

\section{Compressive Radio SPectrum Analysis}

This section describes a system for spectral analysis of a radio spectrum from compressive measurements. We explain the spectrum recovery using a fixed support and contrast it to the use of optimal bases.

\section{A. System Model}

Fig. 1 presents our system model. The objective of the system is to build fine-grain frequency response using compressive sensing. The system employs multiple sensor nodes in parallel that are distributed across a geographical region. Each node senses a portion of a wideband spectrum in time domain and transmits its compressive measurements to the system backend. (The term "system" designates the control entity and "sensor node" an element providing measurements to the system.)

The system partitions the target spectrum into $J$ subbands and dispatches an assignment $\left(f_{i}, B_{i}\right)$ for node $i$; the node tunes to frequency $f_{i}$ (for subband $i$ ) and starts its measurement according to the bandwidth $B_{i}$. The total bandwidth sensed is $B_{t o t}=\sum_{i=1}^{J} B_{i}$. In our model, the base station serves as the data sink to which the compressive measurements are sent first, before delivered to the system backend for recovery.

\section{B. Compressive Sensing with a Fixed Support Set}

Radio signals do not typically reveal their characteristic information in time domain, thus it is customary to look for their frequency responses, for example, using Fourier Transform. We consider Discrete Fourier Transform (DFT) that computes the inner product of an input signal $\mathrm{x}$ with sampled complex sinusoids and yields the frequency response $\mathbf{X}=\mathcal{F} \mathbf{x}$, where $\mathcal{F}$ is the matrix form of DFT with coefficients 


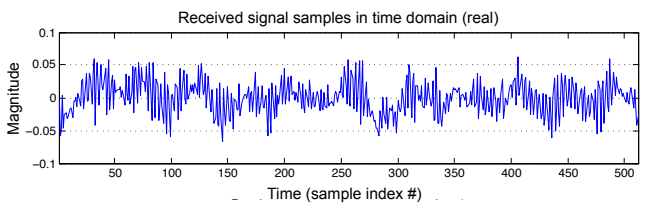

Fig. 2. Time domain representation of an actual wireless channel (source: 512 samples measured using USRP2 from UHF Ch. $21\left(f_{c}=515 \mathrm{MHz}, 6-\mathrm{MHz}\right.$ bandwidth) in Cambridge, MA on Saturday May 21, 2011 23:03 EST)

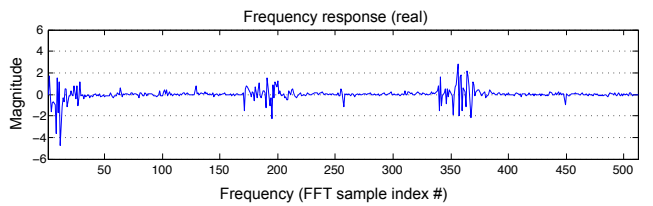

Fig. 3. The samples from Fig. 2 represented using fixed support $\mathcal{F}$

$f_{n k}=e^{-j 2 \pi n k / N}$ for $n, k=0,1, \ldots, N-1 . \mathcal{F}$ is invertible, so we have $\mathbf{x}=\mathcal{F}^{-1} \mathbf{X}$, the Inverse DFT (IDFT).

Using the DFT basis $\mathcal{F}$, we can rewrite the compressive measurements in Eq. (1):

$$
\mathbf{y}=\mathbf{\Phi} \mathbf{x}=\boldsymbol{\Phi}\left(\mathcal{F}^{-1} \mathbf{X}\right)=\left(\mathbf{\Phi} \mathcal{F}^{-1}\right) \mathbf{X}
$$

The key intuition here is that a fixed support $\mathcal{F}$ is used as a sparsifying basis $\boldsymbol{\Psi}$ for compressive sensing decoding. With the matrix product $\mathbf{\Phi} \mathcal{F}^{-1}$, the $\ell_{1}$-minimum decoder can recover $\mathbf{X}$, then $\mathbf{x}$ from $\mathbf{X}$ by IDFT. We use compressive sensing with $\mathcal{F}$ as the baseline approach against which our new approach will be compared.

\section{How Good Is Fourier Basis?}

Fig. 2 illustrates the time-domain representation of the samples measured from an actual UHF channel at its Nyquist rate with $N=512$, using GNU Radio-USRP2 [8], [11]. Evidently, we cannot conclude any sparse structure in the time domain. We take FFT and depict the frequency domain representation of the same samples in Fig. 3. About 23\% of the samples $(\approx 117$ out of 512 samples $)$ are observed to have relatively large magnitudes that can be said nonzero.

\section{Compressive Sensing with Optimal Sparsifying Basis}

Fixed support sets provide signal-independent sparsifying matrix $\boldsymbol{\Psi}$ for decoding, but there is a drawback. Sparsity under a fixed support is not uniform across different subbands, and as a consequence, we should take different number (say, $M_{i}$ for subband $i$ ) of measurements for each subband. This partly motivates the use of an optimal KLT basis for each subband, which makes sparsity across different subbands more uniform and, more importantly for decoding accuracy, also smaller.

In Section II.B, we derived that the KLT basis $\mathbf{Q}$ can give the most compact representation for $\mathbf{x}$ with $\mathbf{s}=\mathbf{Q}^{\mathbf{H}} \mathbf{x}$. In fact, $\mathbf{Q}$ is an ideal candidate for $\boldsymbol{\Psi}$. Similar to Eq. (2), we write compressive sensing with optimal KLT basis:

$$
\mathbf{y}=\mathbf{\Phi} \mathbf{x}=\mathbf{\Phi}(\mathbf{Q s})=(\mathbf{\Phi Q}) \mathbf{s}
$$

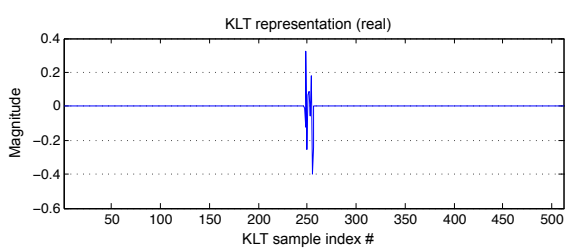

Fig. 4. The samples from Fig. 2 represented using optimal KLT basis

We note that among all $\boldsymbol{\Psi}, \mathbf{Q}$ makes $\mathrm{s}$ with the smallest number of nonzeros. Fig. 4 depicts the KLT representation of the same samples from Fig. 2. We estimated $\mathbf{Q}$ from a sample covariance matrix with 10 previous measurement sets. Compared to the fixed support $\mathcal{F}$, we observed a 14fold gain in sparsity for this example. Such sparsity gain can significantly improve the accuracy of recovery with the same (or even fewer) number of compressive measurements.

\section{RECOVERING KLT BASIS With COMPRESSIVE MEASUREMENTS}

We have mentioned earlier that compressive measurements can be used to recover the KLT matrix. To explain this, we start with the tie-in between the compressive encoding at sensor nodes and KLT basis estimation. Recall that the KLT matrix $\mathbf{Q}$ is computed from the covariance matrix of input signal $\mathbf{x}, \mathbf{R}_{\mathbf{x}}=\mathbb{E}\left[\mathbf{x x}^{\mathbf{H}}\right]$. Similarly, the covariance matrix of the compressive measurements $\mathbf{y}$ is $\mathbf{R}_{\mathbf{y}}=\mathbb{E}\left[\mathbf{y} \mathbf{y}^{\mathbf{H}}\right]$. By compressive encoding $\mathbf{y}=\boldsymbol{\Phi} \mathbf{x}$, we know: $\mathbf{R}_{\mathbf{y}}=\mathbb{E}\left[\boldsymbol{\Phi} \mathbf{x} \mathbf{x}^{\mathbf{H}} \boldsymbol{\Phi}^{\mathbf{T}}\right]=$ $\boldsymbol{\Phi} \mathbb{E}\left[\mathbf{x x}^{\mathbf{H}}\right] \boldsymbol{\Phi}^{\mathbf{T}}$. So, $\mathbf{R}_{\mathbf{y}}=\boldsymbol{\Phi} \mathbf{R}_{\mathbf{x}} \boldsymbol{\Phi}^{\mathbf{T}}$. Note that $\boldsymbol{\Phi}$ is not a square matrix. Using the pseudo-inverse $\left(\boldsymbol{\Phi}^{\mathbf{T}}\right)^{\dagger}$, we can have the following expression:

$$
\mathbf{R}_{\mathbf{y}}\left(\boldsymbol{\Phi}^{\mathbf{T}}\right)^{\dagger}=\mathbf{\Phi} \mathbf{R}_{\mathbf{x}}
$$

Here, we find that we have been compressively measuring $\mathbf{R}_{\mathbf{x}}$ in $\mathbf{R}_{\mathbf{y}}\left(\boldsymbol{\Phi}^{\mathbf{T}}\right)^{\dagger}$, which can be approximated from $\mathbf{y}=\boldsymbol{\Phi} \mathbf{x}$ that we used to encode our data $\mathbf{x}$. Thus compressive measurements $\mathbf{y}$ have sufficient information to recover $\mathbf{R}_{\mathbf{x}}$ from (4). Below is a procedure to estimate KLT basis $\mathbf{Q}$ with compressive measurements in four steps:

1) Decode $\mathbf{X}$ from $\mathbf{y}=\left(\boldsymbol{\Phi} \mathcal{F}^{-1}\right) \mathbf{X}$ using fixed support $\mathcal{F}$;

2) Recover $\mathbf{x}$ by computing $\mathbf{x}=\mathcal{F}^{-1} \mathbf{X}$;

3) Repeat the previous steps $l$ times to numerically compute: $\mathbf{R}_{\mathbf{x}}=\mathbb{E}\left[\mathbf{x x}^{\mathbf{H}}\right]=\frac{1}{l} \sum_{i=1}^{l} \mathbf{x}_{i} \mathbf{x}_{i}{ }^{\mathbf{H}}$

4) Obtain $\mathbf{Q}$ by eigen-decomposition $\mathbf{R}_{\mathbf{x}}=\mathbf{Q} \mathbf{\Lambda} \mathbf{Q}^{\mathbf{H}}$.

\section{IMPLEMENTATION}

\section{A. Workflow and Subsystems}

Fig. 5 outlines the workflow between a sensor node and the system. We faithfully implement a compressive sensing encoder without any modification. Our sensor nodes are implemented in the GNU Radio software framework for USRP2. The nodes perform direct bandpass sampling [12] and convert the analog RF/IF into the complex digital in-phase and quadrature (I-Q) samples. Each sampling instance buffers uncompressed $\mathrm{x}$ containing $N$ I-Q samples, and the encoder uses a pre-generated, $M \times N$ Bernoulli random matrix $\boldsymbol{\Phi}$ to 


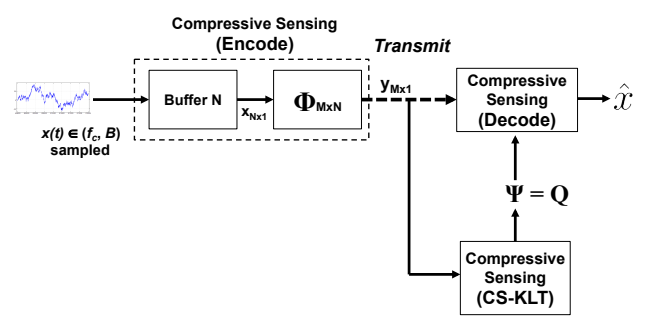

Fig. 5. We implement unmodified compressive sensing encoder but place an additional decoder block, namely CS-KLT, that estimates a KLT basis from the procedure described in Section IV.

produce $M$ compressive measurements before transmitted innetwork. We simulated wireless uplinks to the base station for in-network transmissions.

A novelty in our implementation is in the decoder. We added a new block for KLT basis estimation. It is labeled $C S$ $K L T$ in Fig. 5. CS-KLT is essentially a compressive sensing decoder that recovers an estimate of the covariance matrix from compressive measurements of the input signal. Based on the procedure described in Section IV, we formulated an online algorithm for CS-KLT, explained next.

\section{B. Online Algorithm for CS-KLT}

Algorithm 1 presents our pseudo-code implementation for the CS-KLT online optimal basis estimation. The algorithm aligns to incremental update or complete update duty cycles (tested by a boolean completeUpdate) to determine whether the sample covariance matrix $\mathbf{R}_{\mathbf{x}}$ should be recalculated completely. This reestimate is necessary because in reality wireless channels are time-varying, and stationarity does not persist. Hence, we need to schedule a complete update once every certain number of measurement intervals. In the algorithm, procedure CSKLT invokes CSDECODERX, triggering the system to flush the current $\mathbf{R}_{\mathbf{x}}$ and reacquire an estimate. On a normal measurement interval, procedure CSKLT invokes DOINCUPDATERX. The incremental update is based on weighted averaging (with weight $\alpha$ for $0<\alpha<1$ ) between the two successive intervals (see lines 20-22).

\section{Optimal Interval to Update KLT Basis}

The complete update of $\mathbf{R}_{\mathbf{x}}$ may take up multiple measurement intervals and operate at low compression, costing much more measurements than recovering the input signal on a normal interval. So we should not do complete updates too frequently. An optimal interval for complete updates can be determined from the performance requirement (estimation/decoding errors), stationarity of the channel, and the relative cost of a complete update to an incremental update.

\section{Evaluation}

We evaluated the performance of our CS-KLT against Fourier basis in the following scenarios: (1) spectrum analysis of 200-MHz UHF whitespace; (2) sensing short radio transmissions. We collected all of the data presented in this section empirically from our field and laboratory experiments, using commercially available software radios.

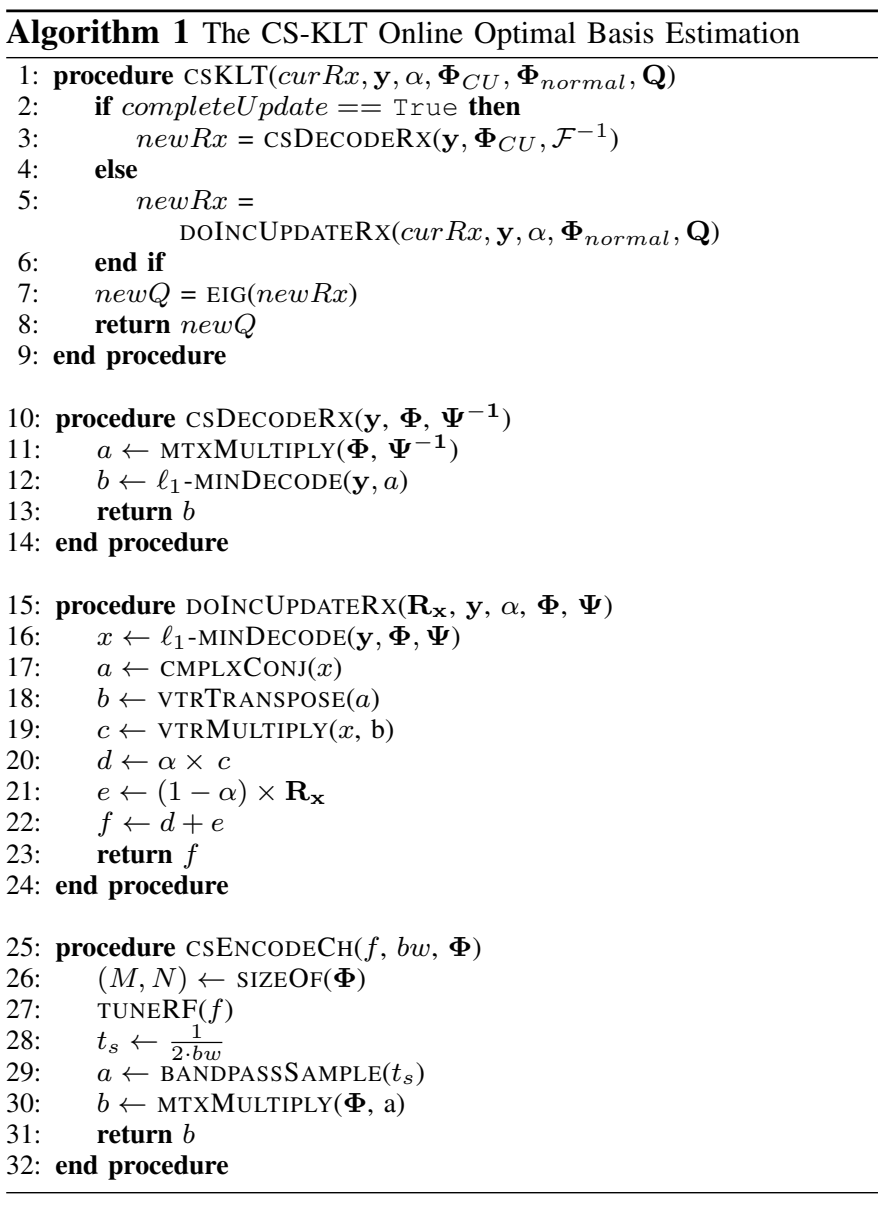

\section{A. Description of Experiments}

1) UHF spectrum analysis: We prepared 4 USRP2 radios in an indoor lab. The spectrum is partitioned into $J=8$ subbands (i.e., each with $25-\mathrm{MHz}$ bandwidth) with center frequencies $f_{c} \in\{512.5,537.5,562.5,587.5,612.5,637.5$, 662.5, 687.5\} MHz. This spans UHF channels 19 to 51. We configured each USRP2 node to alternate between two subbands and measure. Duty cycles were counted in 1-msec unit measurement intervals. The Nyquist rate of each subband is $25 \mathrm{MHz} \times 2=50$ million samples/sec (or 50,000 samples per unit measurement interval). We ran multiple sessions over different days at various hours, with each session lasting up to a minute.

2) Detecting Harris radios: This was conducted in an outdoor wireless test field. We used 4 USRP2 radios for compressive sensing of $100-\mathrm{MHz}$ target spectrum partitioned in $J=4$ subbands. The center frequencies for this experiment were $\{363,386,409,432\} \mathrm{MHz}$, meaning there is a $2-\mathrm{MHz}$ overlap between two adjacent sensors. We placed 4 Harris military radios [13], two of which were engaged in live voice communications in the first subband. The other two were turned on and off randomly in the second and the fourth subbands. No radios transmitted in the third subband. The sessions lasted 10-30 seconds, and we kept other measurement parameters the same as before. 


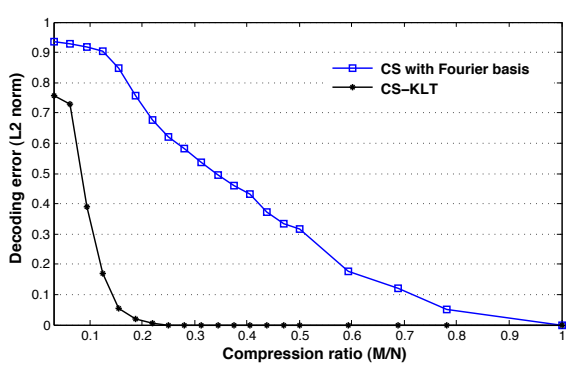

Fig. 6. Error performance on recovering 200-MHz UHF spectrum

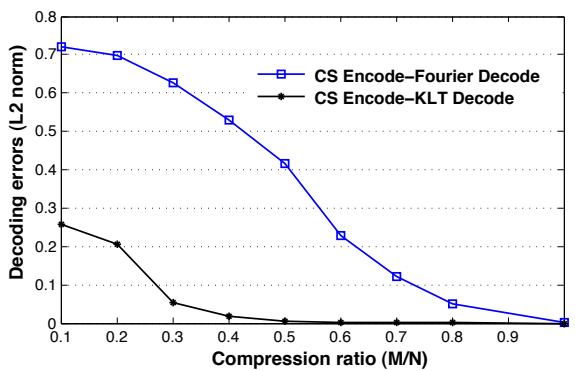

Fig. 7. Error performance on sensing Harris radio transmissions

\section{B. Spectrum Recovery Performance}

1) UHF spectrum: Fig. 6 compares decoding errors of our CS-KLT against Fourier basis under various compression ratios $(M / N)$. For this analysis, we used $N=256$ per subband (resulting 2048-point frequency response for the 200$\mathrm{MHz}$ spectrum). CS-KLT had 100-msec incremental and 1sec complete update duty cycles. We adopted a normalized $\ell_{2}$-norm error metric computed in the frequency domain, $e_{\ell_{2}}=\|\mathbf{X}-\hat{\mathbf{X}}\|_{\ell_{2}} /\|\mathbf{X}\|_{\ell_{2}}$ ( $\hat{\mathbf{X}}$ indicates recovered frequency response). Given a fixed error budget, our method resulted substantial, $2 \mathrm{x}$ to $4.3 \mathrm{x}$ gains in compression. For illustrative purposes, we plot the original frequency response and the recovered frequency responses from compressive measurements in Fig. 9 (Fourier) and in Fig. 10 (KLT).

2) Harris radio sensing: We also observe a clear advantage of CS-KLT in sensing Harris radio transmissions as indicated by the error performances in Fig. 7. The use of KLT basis for this case resulted about $2 \mathrm{x}$ to $3 \mathrm{x}$ gains in compression compared to using Fourier basis for decoding.

\section{Dynamic Identification of Trained Patterns via KLT}

We examined a preliminary application of detecting the $\mathrm{ON} / \mathrm{OFF}$ state combinations of multiple transmitters using previously trained KLT matrices. For this evaluation, we reused the data collected from our Harris radio experiments.

Suppose we wish to detect which of the target transmitters is in the $\mathrm{ON}$ state. To do this, we propose the following target detection strategy based on CS-KLT. First, we perform a training phase, where we estimate the KLT bases for individual Harris radios by activating them one at a time. Let us call the 4 KLT matrices obtained in our field experiment $\mathbf{Q}_{\mathbf{1}}, \ldots, \mathbf{Q}_{\mathbf{4}}$. There could be other ways to obtain the KLT characterizations

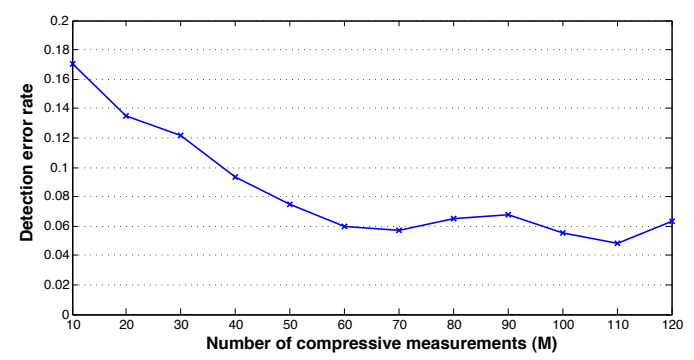

Fig. 8. Error performance of CS-KLT in a real-world experiment

of the targets requiring less access-for example, through training on reference signals or from known signal models such as the power-spectral densities of specific modulation schemes.

Secondly, we perform a separate CS-KLT decoding operation, using each KLT matrix $\mathbf{Q}_{\mathbf{i}}$ as the sparse transform. Each decoding based on $\mathbf{Q}_{\mathbf{i}}$ detects the presence of target $i$, as desired. Clearly, this decoding process can detect any combination of active transmitters. Furthermore, since the KLT bases of individual transmitters do not change, there is no need for periodic updates.

We computed the detection error rate by taking 5-sec long spectrum samples from each of our 4 USRP2s, encompassing 400 blocks of 2048 samples each. We added the samples together into a single stream of measurements and encoded them with compressive sensing. On the decoding, we recovered the combined samples with the KLT matrix $\mathbf{Q}_{\mathbf{3}}$ and used a simple threshold to decide whether Harris radio 3 was $\mathrm{ON}$ or $\mathrm{OFF}$. We present the detection error rate- the fraction of 400 CS-KLT instances in which the decoding decision was incorrect-in Fig. 8 versus the number of measurements $(M)$. We observe that even for small $M$, the error rate is quite low. This is an encouraging outcome, considering that the raw number of samples per measurement interval is close to $10^{4}$.

\section{RELATED WORK}

Polo et al. [5] presented compressive spectrum sensing with distributed cognitive radios, which assumed a similar system model as ours. Wang et al. [14] proposed a compressive sensing decoder that combined analog-to-information converter (AIC) and the SOMP [15] algorithm. Duarte et al. [15] leveraged model-based joint sparsity of wireless signals to improve compressive sensing decoding. Our work is not just about applying classical transforms in the pursuit of optimal sparsifying bases for compressive spectrum sensing, but adaptation to time-variance and nonstationarity, which pose difficult yet most realistic problems. This differentiates our work from others.

\section{CONCLUSION}

In this paper, we described how compressive sensing can benefit from using optimal sparsifying basis. Our approach was based on Karhunen-Loève Transform (KLT) that maximally leverages the correlation structure of a signal. By using an 

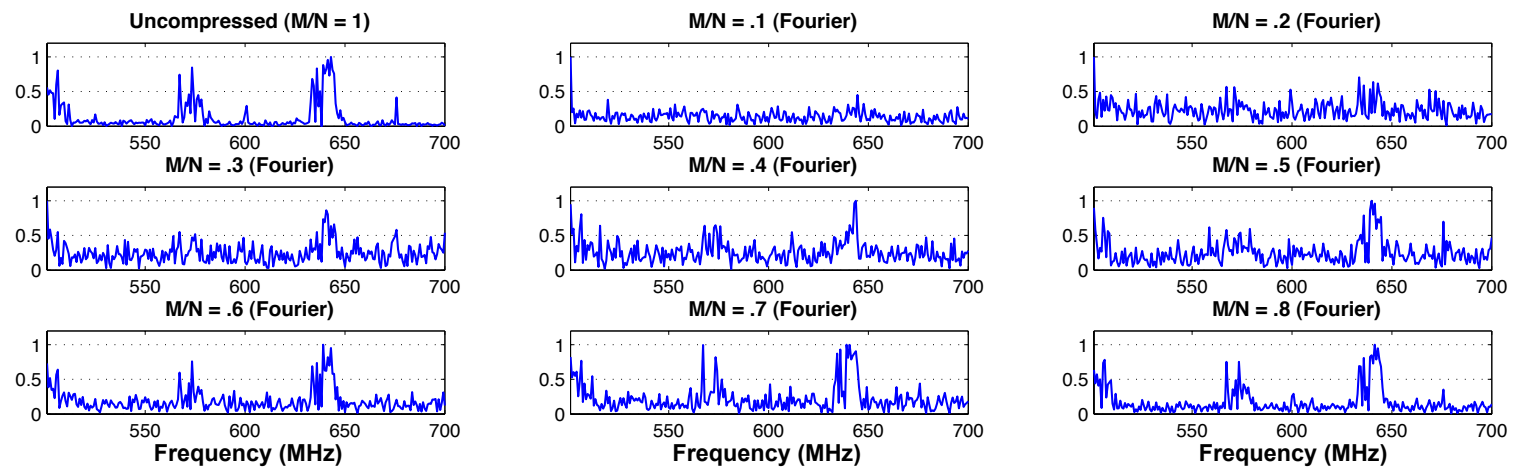

Fig. 9. Recovering 200-MHz UHF spectrum with the Fourier basis under various compression ratios $\left(\frac{M}{N}\right)$
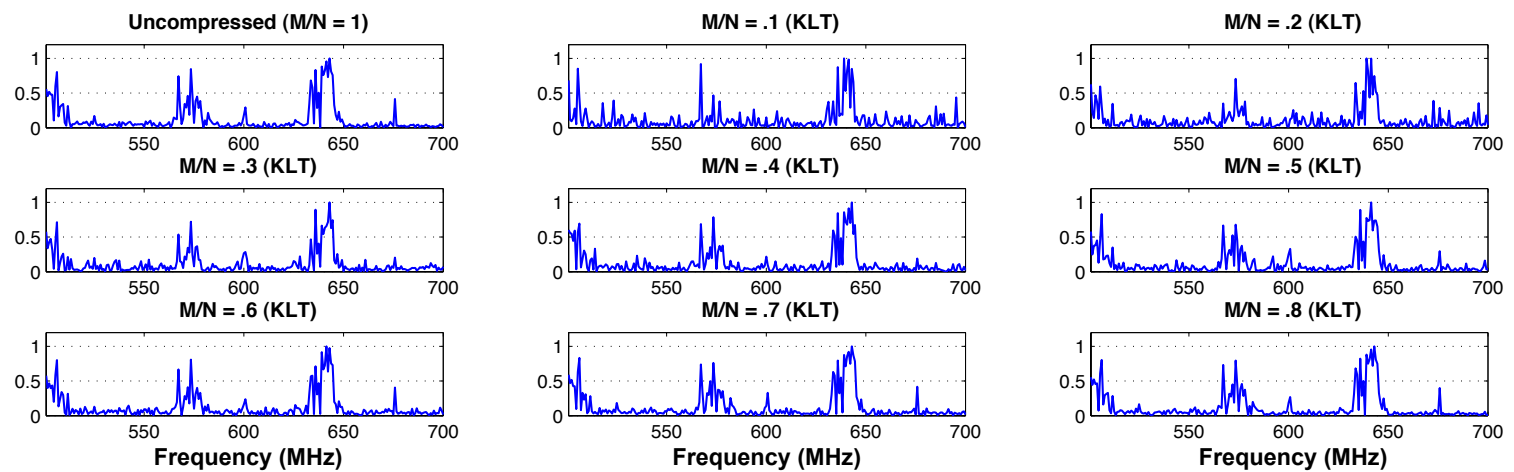

Fig. 10. Recovering 200-MHz UHF spectrum with the KLT basis under various compression ratios $\left(\frac{M}{N}\right)$

optimal KLT basis for decoding, we were able to drastically reduce the number of measurements without affecting the accuracy of data recovery. Our method, highlighted by the CSKLT online algorithm, was designed to work with time-varying wireless channels in reality, updating KLT basis incrementally from compressive measurements. We demonstrated empirical performance gains of our method in spectrum sensing applications. The results of this paper suggested that we can make an effective use of optimal bases in compressive sensing when the data dependency of KLT can be overcome with a proper online updating mechanism. This represents a significant departure from the current practice of compressive sensing applications that almost always uses a fixed support set such as DFT. While encouraged by our results, we realize the need for further work. It would be useful to develop a comprehensive theory on the efficiency limit of optimal basis compressive sensing. For the time being, we recover the covariance matrix with DFT basis, but other estimation method could be used for this purpose. Lastly, we plan to explore applications that leverage the new opportunity enabled by optimal basis compressive sensing.

\section{ACKNOWLEDGMENT}

This material is in part based on research sponsored by Air Force Research Laboratory under agreement number FA8750-10-2-0180. The U.S. Government is authorized to reproduce and distribute reprints for Governmental purposes notwithstanding any copyright notation thereon. The views and conclusions contained herein are those of the authors and should not be interpreted as necessarily representing the official policies or endorsements, either expressed or implied, of Air Force Research Laboratory or the U.S. Government.

\section{REFERENCES}

[1] E. J. Candès and T. Tao, "Decoding by Linear Programming," IEEE Trans. on Information Theory, vol. 51, no. 12, pp. 4203-4215, 2005.

[2] C. Luo, F. Wu, J. Sun, and C. W. Chen, "Compressive data gathering for large-scale wireless sensor networks," in ACM MOBICOM, 2009.

[3] H. T. Kung, C.-K. Lin, and D. Vlah, "CloudSense: Continuous Finegrain Cloud Monitoring with Compressive Sensing," in USENIX HotCloud, 2011.

[4] Z. Tian and G. Giannakis, "Compressed Sensing for Wideband Cognitive Radios," in IEEE ICASSP, 2007.

[5] Y. Polo, Y. Wang, A. Pandharipande, and G. Leus, "Compressive Wideband Spectrum Sensing," in IEEE ICASSP, 2009.

[6] Y. Gwon, H. T. Kung, and D. Vlah, "DISTROY: Detecting IC Trojans with Compressive Measurements," in USENIX HotSec, 2011.

[7] K. Karhunen, "Über Lineare Methoden in der Wahrscheinlichheitsrechnung," PhD Dissertation, University of Helsinki, 1947.

[8] The Universal Software Radio Peripheral (USRP) Products, Ettus Research LLC. [Online]. Available: http://www.ettus.com/

[9] E. J. Candès, J. Romberg, and T. Tao, "Robust Uncertainty Principles," IEEE Trans. on Information Theory, vol. 52, no. 2, pp. 489-509, 2006.

[10] E. J. Candès, "Compressive Sampling," in International Congress of Mathematicians, 2006.

[11] GNU Radio. [Online]. Available: http://gnuradio.org

[12] W. Waters and B. Jarrett, "Bandpass Signal Sampling and Coherent Detection," IEEE Trans. on Aerospace and Electronic Systems, vol. 18, no. 6, pp. 731-736, 1982.

[13] Harris Radio. [Online]. Available: http://www.harris.com/

[14] Y. Wang, A. Pandharipande, Y. Polo, and G. Leus, "Distributed Compressive Wide-band Spectrum Sensing," in ITA Workshop, 2009.

[15] M. Duarte, S. Sarvotham, D. Baron, M. Wakin, and R. Baraniuk, "Distributed Compressed Sensing of Jointly Sparse Signals," in Signals, Systems and Computers, 2005. 